\title{
Prediction of Low-Dose Aspirin-Induced Gastric Toxicity Using Nuclear Magnetic Resonance Spectroscopy-Based Pharmacometabolomics in Rats
}

\author{
Abubakar Sha'aban 1,2, , Hadzliana Zainal 1,", Nor Azlina Khalil ${ }^{3}$, Fatimatuzzahra' Abd Aziz ${ }^{1}$, Ewe Seng Ch'ng ${ }^{3}$, \\ Chin-Hoe Teh ${ }^{4}$, Mustapha Mohammed ${ }^{1,2,}$, Baharudin Ibrahim 1,5,*
}

1 School of Pharmaceutical Sciences, Universiti Sains Malaysia, Penang, 11800, Pulau Pinang, Malaysia; abuushaaban2@gmail.com; hadz@usm.my; faa@usm.my; mohammedmmrx@gmail.com; baharudin.ibrahim@um.edu.my

2 Department of Clinical Pharmacy and Pharmacy Practice, Faculty of Pharmaceutical Sciences, Ahmadu Bello University, Zaria, Kaduna, Nigeria

3 Advanced Medical and Dental Institute, Universiti Sains Malaysia, Bertam, 13200, Kepala Batas, Pulau Pinang, Malaysia; norazlinakhalil@usm.my

4 Bruker (Malaysia) Sdn Bhd, Bayan Lepas, Malaysia; chin_hoe.teh@bruker.com

5 Faculty of Pharmacy, Universiti Malaya, Kuala Lumpur, 50603, Malaysia

* Correspondence: abuushaaban2@gmail.com (AS); hadz@usm.my (HZ); mohammedmmrx@gmail.com (MM); baharudin.ibrahim@um.edu.my (BI)

\begin{abstract}
Background: Low-dose aspirin (LDA) is the backbone for secondary prevention of coronary artery disease, though limited by gastric toxicity. This study was aimed to identify novel metabolites that could predict LDA-induced gastric toxicity using pharmacometabolomics. Methods: Pre-dosed urine samples were collected from male Sprague-Dawley rats. The rats were treated with either LDA $(10 \mathrm{mg} / \mathrm{kg})$ or $1 \%$ methylcellulose $(10 \mathrm{ml} / \mathrm{kg})$ per oral for 28 days. The rats' stomachs were examined for gastric toxicity using a stereomicroscope. The urine samples were analyzed using a proton nuclear magnetic resonance spectroscopy. Metabolites were systematically identified by exploring established databases and multivariate analyses to identify the spectral pattern of metabolites related to LDA-induced gastric toxicity. Results: Treatment with LDA resulted in gastric toxicity in 20/32 rats (62.5\%). The orthogonal projections to latent structures discriminant analysis (OPLS-DA) model displayed a goodness-of-fit $\left(R^{2} Y\right)$ value of 0.947 , suggesting a near-perfect reproducibility, a goodness-of-prediction $\left(\mathrm{Q}^{2} \mathrm{Y}\right)$ of -0.185 with perfect sensitivity, specificity and accuracy $(100 \%)$. Furthermore, the area under the receiver operating characteristic (AUROC) displayed was 1. The final OPLS-DA model had an $\mathrm{R}^{2} \mathrm{Y}$ value of 0.726 and $\mathrm{Q}^{2} \mathrm{Y}$ of 0.142 with sensitivity $(100 \%)$, specificity (95.0\%) and accuracy (96.9\%). Citrate, hippurate, methylamine, trimethylamine $\mathrm{N}$-oxide and alpha-keto-glutarate were identified as the possible metabolites implicated in the LDA-induced gastric toxicity. Conclusion: The study identified metabolic signatures that correlated with the development of a low dose Aspirin-induced gastric toxicity in rats. This pharmacometabolomic approach could further be validated to predict LDA-induced gastric toxicity in patients with coronary artery disease.
\end{abstract}

Keywords: aspirin; pharmacometabolomic; nuclear magnetic resonance; spectroscopy; gastric toxicity; multivariate analysis

\section{Introduction}

Coronary artery disease (CAD) is a leading cause of cardiovascular disease (CVD) related morbidity and mortality globally [1]. Low-dose aspirin (LDA) is the mainstay for the secondary prevention of CAD [2]. Aspirin inhibits platelet activity by irreversibly deactivating cyclooxygenase-I (COX-1), leading to inhibition of platelet thromboxane-A2 
(TXA2) production, TXA2-mediated platelet activation [3]. The activity of Aspirin on TXA2 explains its distinct efficacy in preventing atherothrombosis and shared gastrointestinal (GI) side effects with other antiplatelets [3]. Alternative antiplatelets or co-administration with gastro-protective agents are presently the most common strategies to reduce Aspirin-induced GI side effects [4,5]. However, alternative Aspirin use is limited with cost burden, pill burden, and decreased effectiveness, necessitating the need for more cost-effective strategies.

There are limited studies on strategies, like pharmacometabonomics, to predict the manifestation of gastric toxicity prior to LDA dosing. Pharmacometabolomics is a fast, economic and less invasive approach to predict drug-induced toxicity and complement personalized therapy. The proton nuclear magnetic resonance ( $\left.{ }^{1} \mathrm{H}-\mathrm{NMR}\right)$ spectroscopy is a relatively new methodology for predicting drug effects using pre-dosed biomarkers of biofluids. The NMR spectroscopy-based pharmacometabolomics is defined as "the prediction of the outcome (e.g., toxicity or efficacy) of a drug or xenobiotic in individuals, based on a mathematical model of pre-intervention metabolite signatures" [6]. The NMR spectroscopy is the "gold standard" in pharmacometabolomics because of its non-destructive nature and enables observation of the dynamics, partition and quantification of metabolites in bio-samples. The pharmacometabolomics metabolomics combines 1H-NMR and multivariate analysis, to provide a detailed examination of the changes in the metabolic signatures of a bio-samples. Therefore, this study was aimed to identify metabolites that predict LDA-induced gastric toxicity using ${ }^{1} \mathrm{H}-\mathrm{NMR}$-based pharmacometabonomics in rats.

\section{Results}

\subsection{Gastric toxicity}

At the end of the low dose aspirin dosing period (28 days), none of the rats $0 / 10(0 \%)$ in the vehicle-treated group had any form of gastric toxicity. However, most rats 20/32 $(62.5 \%)$ in the LDA-treated group developed gastric toxicity.

\subsection{Pre-dose profiling models}

The Orthogonal Projections to Latent Structures Discriminant Analysis (OPLS-DA) score plots for the profiling model displayed clear discrimination between the gastric toxic and non-toxic groups, as shown in Figure 1 . The model had a goodness-of-fit value $\left(R^{2} Y\right)$ of 0.947 (very close to 1 ). However, the goodness-of-prediction value $\left(\mathrm{Q}^{2} \mathrm{Y}\right)$ of $-0.185(<0.4)$ indicates that the model has a poor predictive capacity.

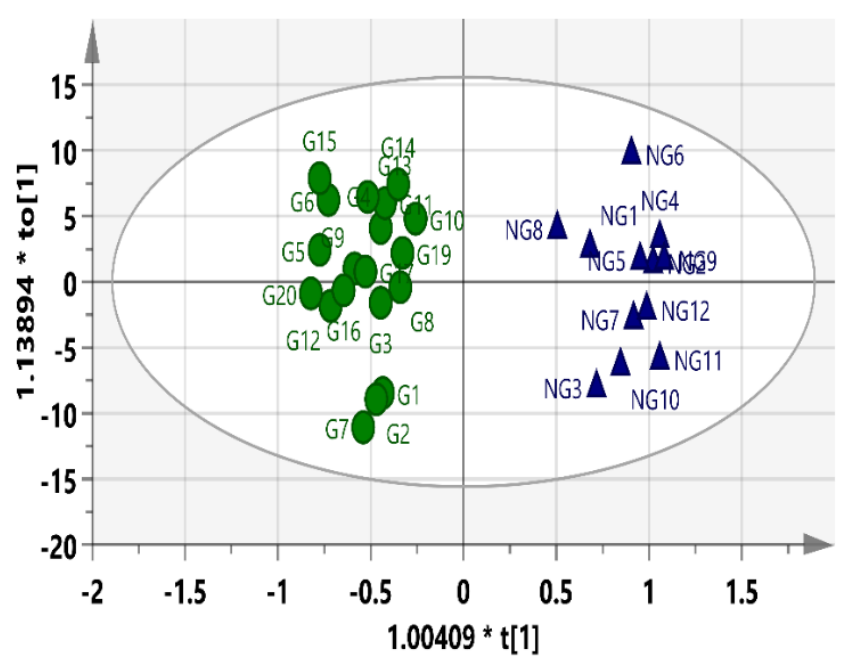

Figure 1. OPLSDA score plots for the profiling model discriminations Blue triangles $=$ Non-gastric toxic rats; Green circles $=$ Gastric toxic rats 
The model has perfect (100\%) sensitivity, specificity, and accuracy values. It also has a perfect AUROC curve value of 1 . The permutation test gave an $R^{2} Y$ intercept value of 0.919 and $\mathrm{a} \mathrm{Q}^{2} \mathrm{Y}$ intercept value of -1.02. There is an overlap between the red and blue lines of the AUROC curve because the AUC value is 1 in both cases (Figure 2).
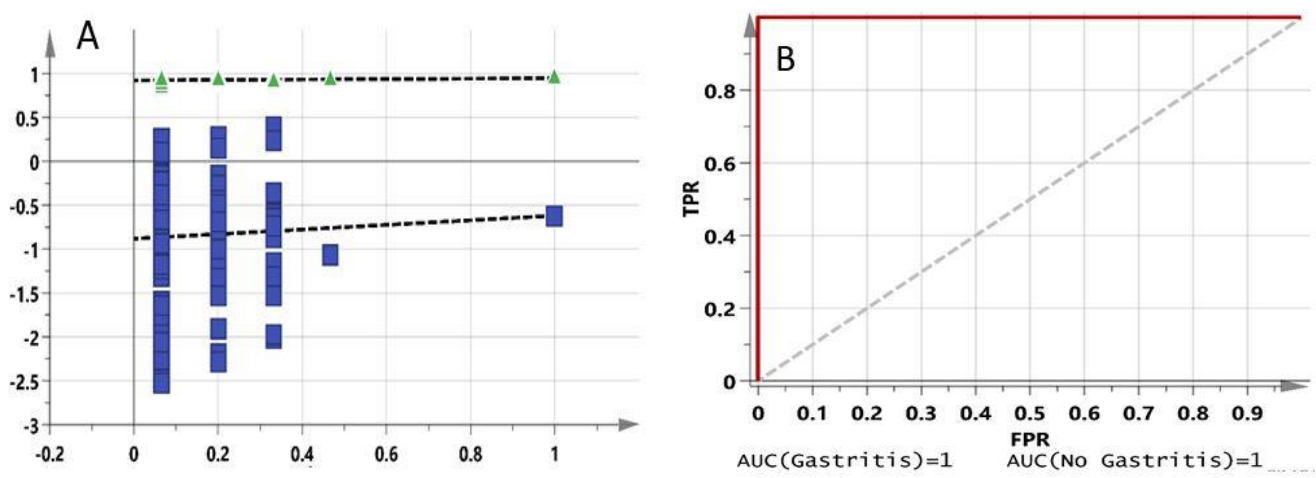

Figure 2. (A) Permutation plot for pre-dose rat urine profiling model. (B) AUROC curve for pre-dose rat urine fingerprinting model.

The number of variables with VIP value $>1$ was 72 , as highlighted in Table 1 . Further examination and exclusion of spectral noise using Topsin resulted in 10 regions ascertained as signals integrated with Topspin before uploading to SIMCA for further screening and identifying useful discriminating metabolites.

Table 1. Summary of pre-dose urine parameters for profiling rat model

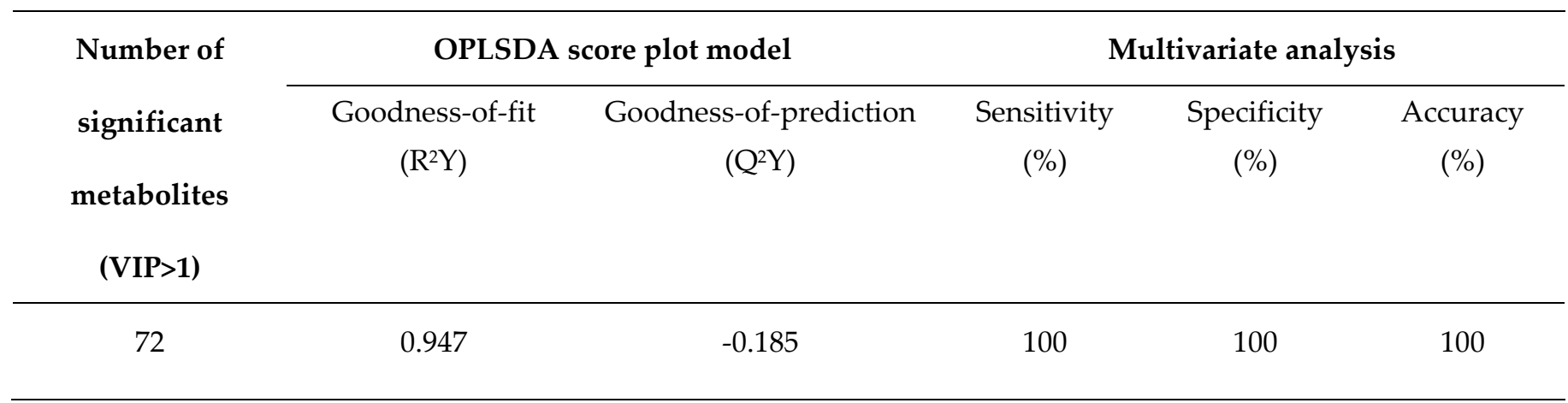

\subsection{Pre-dosed identification models}

Except for two gastric toxic samples (G12 and G3) misclassified to be in the non-gastric toxic group, the pre-dose urine model successfully segregated the samples into gastric toxic and non-toxic groups, as shown in Figure 3. 


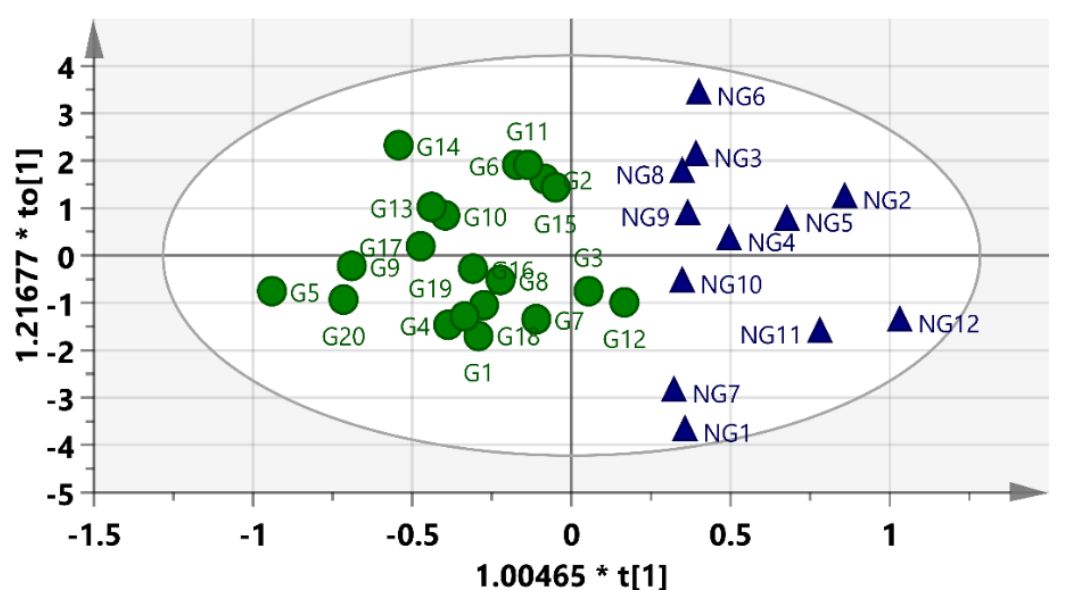

Figure 3. OPLS-DA score plot of pre-dose rat urine identification model

The goodness values are also summarized in Table $2 . R^{2} Y$ value was 0.726 , and the $\mathrm{Q}^{2} \mathrm{Y}$ value was 0.142 . The sensitivity, specificity and accuracy of the identification model were $100 \%, 95 \%$ and $96.88 \%$, respectively.

Table 2. Summary of pre-dose urine parameters for identification model

\begin{tabular}{cccccc}
\hline \multirow{2}{*}{$\begin{array}{c}\text { Number of } \\
\text { significant } \\
\text { metabolites }\end{array}$} & \multicolumn{2}{c}{ OPLSDA score plot model } & \multicolumn{3}{c}{ Multivariate analysis } \\
\cline { 2 - 6 }$(\mathrm{VIP}>\mathbf{1})$ & $\left(\mathrm{R}^{2} \mathrm{Y}\right)$ & $\left(\mathrm{Q}^{2} \mathrm{Y}\right)$ & $\begin{array}{c}\text { Gensitivity } \\
(\%)\end{array}$ & $\begin{array}{c}\text { Specificity } \\
(\%)\end{array}$ & $\begin{array}{c}\text { Accuracy } \\
(\%)\end{array}$ \\
\hline 10 & & & & & \\
\hline
\end{tabular}

The model also has an AUROC value of 1 . Further, the model was internally validated using the permutation plot Figure 4 . The pre-dose rat urine identification model passed all the validity criteria plot. The Q2 values to the left are lower than the original $\mathrm{Q} 2$ point on the right. The blue regression line of the $\mathrm{Q} 2$ point intersects the vertical axis (on the left) below 0 (-0.875). Also, all the R2 values (to the left) are lower than the original $\mathrm{R}^{2}$ value.
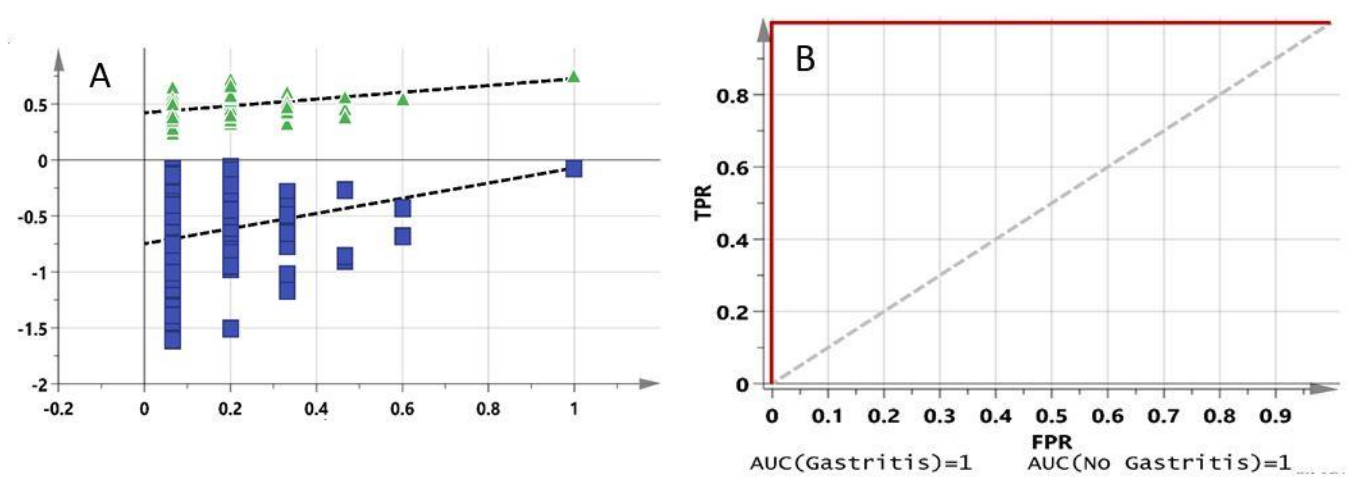

Figure 4. (A). Permutation plot for pre-dose rat urine identification model. (B). AUROC curve (right) for pre-dose rat urine identification model

2.4. Identification of biomarkers to predict $L D A$-induced gastric toxicity 
After the searches in the three databases, the ten regions of the pre-dose rats' urine were identified to correspond to 5 metabolites, as highlighted in Table 3 . These five metabolites were identified as putative biomarkers that may predict LDA-induced gastric toxicity.

Table 3. Potential pre-dose urine metabolites that may predict gastric toxicity

\begin{tabular}{llll}
\hline Chemical shift & Multiplicity & Compound & Molecular formula \\
\hline $2.718-2.677$ & Doublet & Citrate & $\mathrm{C}_{6} \mathrm{H}_{5} \mathrm{O}_{7}{ }^{3}$ \\
$2.571-2.531$ & Doublet & Citrate & $\mathrm{C}_{6} \mathrm{H}_{5} \mathrm{O}_{7}{ }^{-3}$ \\
$2.70-2.680$ & Singlet & Methylamine & $\mathrm{CH}_{3} \mathrm{NH}_{2}$ \\
$3.284-3.277$ & Singlet & Trimethylamine $\mathrm{N}-\mathrm{Oxide}$ & $\mathrm{C}_{3} \mathrm{H}_{9} \mathrm{NO}$ \\
$3.981-3.965$ & Doublet & Hippurate & $\mathrm{C}_{9} \mathrm{H}_{9} \mathrm{NO}_{3}$ \\
$7.571-7.539$ & Triplet & Hippurate & $\mathrm{C}_{9} \mathrm{H}_{9} \mathrm{NO}_{3}$ \\
$7.850-7.825$ & Doublet & Hippurate & $\mathrm{C}_{9} \mathrm{H}_{9} \mathrm{NO}_{3}$ \\
$7.656-7.626$ & Triplet & Hippurate & $\mathrm{C}_{9} \mathrm{H}_{9} \mathrm{NO}_{3}$ \\
$2.459-2.431$ & Triplet & Alpha-keto-glutarate & $\mathrm{C}_{5} \mathrm{H}_{6} \mathrm{O}_{5}$ \\
$3.024-2.996$ & Triplet & Alpha-keto-glutarate & $\mathrm{C}_{5} \mathrm{H}_{6} \mathrm{O}_{5}$ \\
\hline
\end{tabular}

An example of the spectral differences in the metabolites identified between the gastric toxic and non-gastric toxic rats is depicted in Figure 5. The triplet at 2.431-2.459 ppm belonging to alpha-keto-glutarate, doublet at 2.531-2.571 ppm belonging to citrate and a doublet at 3.965-3.981 ppm belonging to hippurate are used to show the differences. 


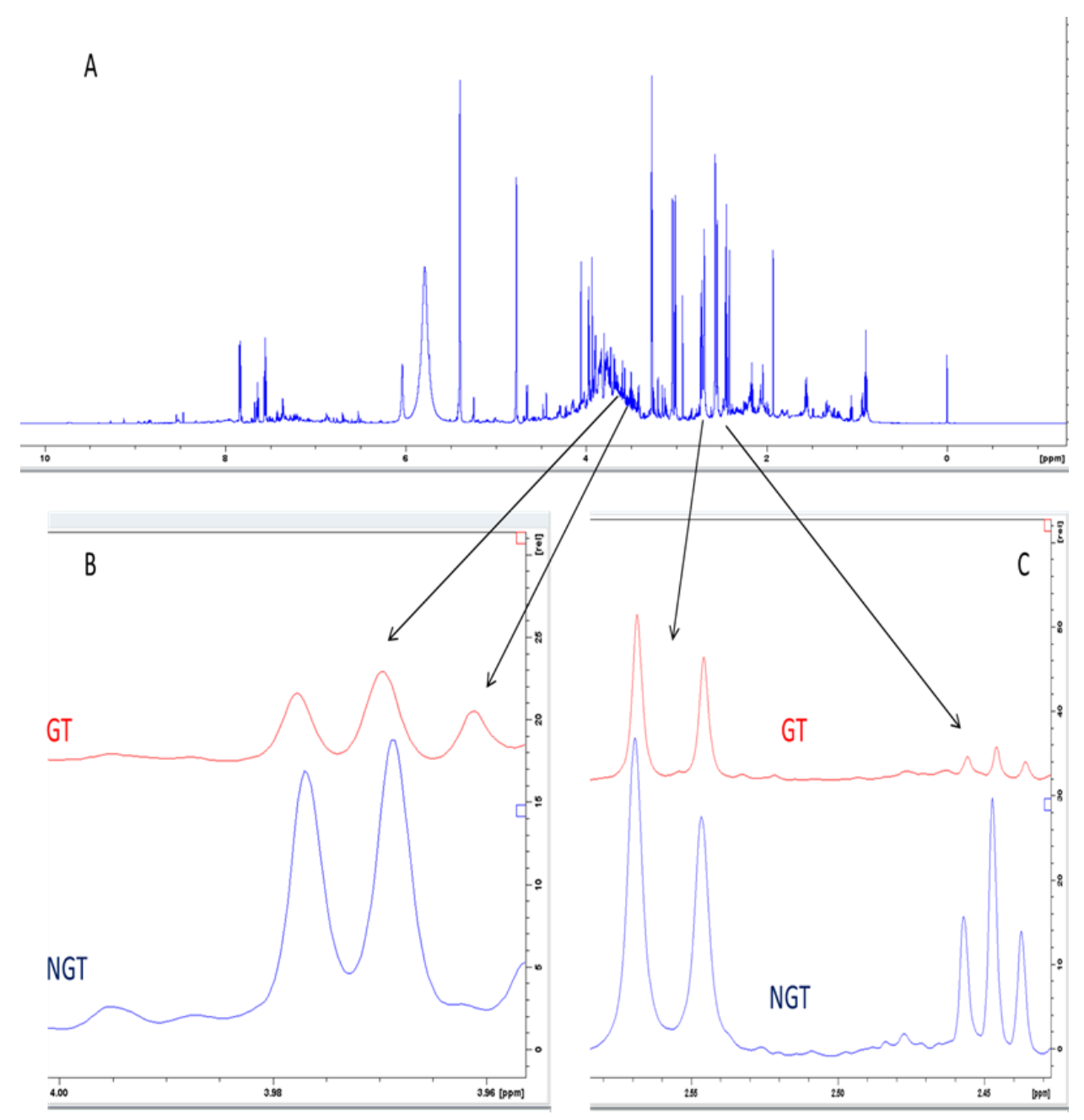

Figure 5. Example of spectral differences between gastric toxic (GT) and non-gastric toxic (NGT) rats

\section{Discussion}

The study demonstrated the robustness of the model developed for pre-dosed prediction of LDA-induced gastric toxicity. The model reaffirms the assertion by Szymańska and colleagues that the area under the receiver operating characteristic (AUROC) or the number of misclassification is more precise in detecting biomarkers responsible for 2-class differentiation or discrimination [19]. They also proclaim that $\mathrm{Q}^{2}$ values are not very good as diagnostic statistics in discriminatory analysis models like orthogonal projections to latent structures discriminant analysis (OPLS-DA) [19]. The goodness-of-fit $\left(R^{2} Y\right)$ value of 0.947 indicates the near-perfect reproducibility of the model. However, the goodness-ofprediction $\left(\mathrm{Q}^{2} \mathrm{Y}\right)$ value of -0.185 (less than the recommended 0.4 for biological models) shows that the model has poor predictive capacity [20]. The goodness-of-prediction values even lower than 0.3 have been reported in several metabolomic studies [21, 22]. In such instances, it is recommended that the models are further assessed using permutation tests $[22,23]$. Validation by permutation test displayed a near excellent discriminatory ability of the model based on the highlighted parameters by previous researchers [19, 24].

Internal validation through a permutation test indicates no overfitting in the model. It indicates that the model is non-spurious. The AUROC value of 1 further confirms the validity of the model. Based on these statistical parameters, this model is suitable for predicting LDA-induced gastric toxicity. Previous researchers stated that models with good sensitivity and specificity are suited for both screening and confirmation of disease [25]. 
Therefore, the diagnostic statistics qualify the model for developing a diagnostic kit, which can be used clinically to screen patients with the propensity to develop LDA-induced gastric toxicity when validated in a human study. Logically, a minimal number of discriminating metabolites will promote their clinical acceptance and utility. Reducing the crucial bins from 72 to 10 makes the model more clinically relevant. The four identified metabolites (corresponding to 8 out of the ten significant bins) from the final model may be used to develop a diagnostic kit that can be clinically useful.

The citric acid (citrate) is a weak acid formed endogenously in the tricarboxylic acid (TCA) cycle or consumed through some foods. The TCA cycle is also known as the citric acid cycle. NSAIDs have been found to cause the opening of "mitochondrial permeability transition pore", consequently leading to the uncoupling of oxidative phosphorylation, increasing the resting state respiration and disrupting the mitochondrial transmembrane potential. These NSAID-induced changes play a significant function in initiating tissue damage [26]. Takeuchi and colleagues [26] found no changes in serum citrate concentration after administering NSAIDs, including aspirin. They, however, found a decrease in citrate levels in stomach tissue extracts compared with controls. They, therefore, deduced two events to be associated with NSAID-induced gastric injury, viz; hyperactivity of collagenase in the stomach and a decrease in levels of citrate (and other metabolites) as indicators of altered TCA cycle activity, which is a mitochondrial pathway. Other researchers [27], found citrate to be statistically reduced in the NSAID-induced gastric damage group compared to control. Also, Takeuchi, et al. [28] reported no change in serum levels of citrate after low-dose aspirin.

Methylamine is an endogenous metabolite resulting from the breakdown of amine. Its tissue level is found to rise in some disease conditions like diabetes mellitus. The levels of methylamine and ammonia are mutually controlled by a multi-functional enzyme known as semicarbazide-sensitive amine oxidase (SSAO). The activity of SSAO deaminates methylamine to formaldehyde, thereby producing ammonia and hydrogen peroxide. A rise in serum SSAO activity has been reported in patients with some disease conditions, including diabetes mellitus, Alzheimer's disease and vascular disorders. Deamination of methylamine catalyzed by SSAO leads to the production of toxic formaldehyde. In this study, methylamine is one of the predictors of LDA-induced gastric toxicity in the urine. The exact mechanism/pathway linking aspirin-induced GI toxicity and methylamine has not been established. Perhaps future studies can focus on identifying the links which may show other pathways related to aspirin-induced GI toxicity.

Trimethylamine N-oxide (TMAO) results from the oxidation of trimethylamine. It is a common metabolite in both humans and animals. Specifically, TMAO is endogenously synthesized from trimethylamine derived from choline. Choline is usually obtained from either dietary lecithin or carnitine [29]. A link between blood and urinary levels of TMAO and gut microbiota has been established [30]. The concentration of TMAO increases if the number of bacteria that convert the trimethylamine to TMAO in the gut increases. It can, therefore, be inferred from this that those subjects having a higher number of microbes that promote the synthesize of TMAO are also at an increased risk of developing aspirininduced gastric toxicity. Previous researchers reported that NSAIDs increase the level of TMAO compared to controls [27].

Hippuric acid is a product of the conjugation of benzoic acid with glycine. It is referred to as an acyl glycine. Acyl glycines are synthesized through an enzyme known as glycine $\mathrm{N}$-acyl transferase. Hippuric acid is a common constituent of urine, and its quantity is increased with an increase in the intake of phenolic compounds like tea, fruit juices and wine. These phenols are changed to benzoic acid, which is subsequently converted to hippuric acid and excreted in the urine. Gastrointestinal microflora appears to be responsible for quinic acid metabolism into hippuric acid. Indomethacin has been found to cause a decrease in hippurate, possibly due to disruption of the normal microorganisms in the gastrointestinal tract [31].

One of two ketone derivatives of glutaric acid is alpha-ketoglutaric acid, also known as 2-oxoglutaric acid. When used without qualification, the word "ketoglutaric acid" 
usually always refers to the alpha version. The only difference between beta ketoglutaric acid and other ketoglutaric acids is the position of the ketone functional group, and it is significantly less prevalent. Alpha-ketoglutarate, commonly known as 2-oxoglutarate, is a biologically significant carboxylate. It's a keto acid formed when glutamate is deaminated, and it's an intermediate in the Krebs cycle [32].

\section{Materials and Methods}

\subsection{Animals}

Male Sprague-Dawley (SD) rats (250-300 g body weight) were obtained from the Animal Research Complex (ARC) of Advanced Medical and Dental Institute (AMDI), Universiti Sains Malaysia and acclimatized to the animal research room for seven days. The rats were given access to Altromin-1320 maintenance diet for rats/mice (Altromin International, Germany) and water ad libitum. The rats were housed in a room maintained at a temperature of $20 \pm 2 \circ \mathrm{C}$, relative humidity of $55 \pm 10 \%$ respectively, and a 12-hr light/dark cycle throughout the study. The rats were kept in individual cages (1 rat per cage) when samples were not taken but placed in metabolic cages during the periods for sample collection. The experimental protocol was approved by the Institutional Animal Care and Use Committees (IACUC). All procedures were carried out according to recommendations of the IACUC.

\subsection{Experimental protocol}

Two experimental groups were designed using a stratified randomization system based on rats' bodyweight viz: group I (control, $n=10$ ) and group II (treatment, $n=32$ ). The rats in group I were administered $1 \%$ methylcellulose $(10 \mathrm{ml} / \mathrm{kg})$, while those in group II were administered low-dose aspirin, LDA $(10 \mathrm{mg} / \mathrm{kg})$ per oral for 28 days through the intra-gastric (IG) route with an oral gavage. Aspirin, sparingly soluble in water, was suspended in $1 \%$ methylcellulose before administration. The LDA $(10 \mathrm{mg} / \mathrm{kg})$ was equivalent to the clinical dose of $100 \mathrm{mg}$ daily for adults $[7,8]$ and as used in a similar study to demonstrate LDA-induced gastric toxicity in rats [8].

\subsection{Sample collection}

The rats were transferred to individual metabolic cages three days before urine collection to acclimatize before the sample collection [9]. Twenty-four-hour urine samples were collected on day 1 (pre-dosed) and day 28 (post-dosed) using the metabolic cage urine collector containing a preservative $(0.5 \mathrm{ml}$ of $100 \mathrm{mg} / \mathrm{ml}$ solution of sodium azide (NaN3)) [6]. The amount of each urine sample received was recorded, transferred into a $15 \mathrm{ml}$ falcon tube, centrifuged at $2500 \mathrm{~g}$ for $10 \mathrm{mins}$ at $4^{\circ} \mathrm{C}$ to remove particles [10], and aliquoted into two $2 \mathrm{ml}$ microcentrifuge tubes. The aliquot and the remaining bulk of urine were stored in a $-80^{\circ} \mathrm{C}$ freezer until analysis by the proton nuclear magnetic resonance (1H-NMR) spectroscopy. All rats were euthanized on the 28th day of the experiment with an overdose of ketamine/xylazine $(91.0 / 9.1 \mathrm{mg} / \mathrm{ml})$ cocktail.

\subsection{Stomach preparation}

Four millilitres of $10 \%$ aqueous buffered formalin was instilled IG using oral gavage for in situ intraluminal fixations to preserve the integrity of the stomach tissue before opening up the abdominal cavity for stomach harvesting [11]. The stomachs were detached after five minutes in situ fixation and excised along the greater curvature. They are subsequently rinsed with cold saline and pinned on a polystyrene board with the mucosa facing upward to flatten the stomach. After that, the stomachs were dried using a manual blower. Drying was necessary to enhance the sample visualization and prevent the light reflection from the microscope.

Each stomach sample was examined under the stereomicroscope (SZ61, Olympus Europa Holding GMBH, Germany). The software (Cellsens) was launched on a desktop monitor, and the stomach images were captured. The image of the whole stomach could 
not be captured at once, even at the lowest zoom magnification (0.67X) and working distance $(110 \mathrm{~mm})$, hence segments were snapped and later stitched into a single image using Photoshop (Adobe Photoshop CS5 Version: 12.0) as recommended by the pathologist. The ulcerations and, likewise, the whole glandular stomach perimeter were measured with the aid of CellSens life science imaging software (Ver. 1.9 Olympus America, Inc.). The stomachs were primarily classified based on the presence or absence of lesions (gastric toxicity).

\subsection{Pharmacometabolomics analysis}

The 1H-NMR spectra were acquired at 700.14 MHz using an ASCEND ${ }^{\mathrm{TM}} 700 \mathrm{MHz}$ NMR Spectrophotometer (Bruker BioSpin Corp., Germany). A day preceding the NMR analysis, aliquots of the urine samples to be analyzed were transferred from the freezer ($8^{\circ} \mathrm{C}$ ) into the refrigerator $\left(4^{\circ} \mathrm{C}\right)$ and allowed to thaw overnight (at least 8 hours) to avoid sample degradation resulting from abrupt defrosting [12]. The thawed aliquots of the samples were centrifuged (MIKRO 22 R, HettichZentrifugen ${ }^{\circledR}$, Germany) at 12,000 g at $4^{\circ} \mathrm{C}$ for 5 minutes to remove any insoluble sediment from the solution. A $400 \mu \mathrm{l}$ of urine and $200 \mu \mathrm{l}$ of phosphate buffer were mixed (2:1) in a microcentrifuge tube [13], and vortexed for a few seconds to ensure uniform mixing. Then, $550 \mu \mathrm{l}$ was transferred to a $5 \mathrm{~mm}$ NMR tube using a pipette and securely closed by its cap (BRUKER®, BioSpin, Germany).

To ensure that an NMR tube was properly positioned, a sample gauge was used to align the NMR tube in the spinner. The tubes were then inserted into the respective sample holders and loaded into the spectrometer using Bruker's IconNMR ${ }^{\mathrm{TM}}$ automation software. After insertion of the NMR tubes, the spectra of the ${ }^{1} \mathrm{H}-\mathrm{NMR}$ were acquired and processed using the automation interphase of IconNMR ${ }^{\mathrm{TM}}$ with TopSpin 3.5 (BRUKER®, BioSpin, Germany) software. The acquisition stages were shimming, locking, baseline correction and phase correction. All spectra were acquired without spinning the sample. Each sample is given a lag time of five minutes (300 s) for thermal equilibration in the magnetic field before measuring $300 \mathrm{~K}$. For each sample, the probe was automatically tuned, matched, the magnetic field was locked on Urine+D2O, and shimmed through a specifically optimized shim file for urine samples. Automatic $1 \mathrm{H}$ pulse calibration (pulsecal) was performed on each sample to reduce sample variability effects due to salt contents. The 1H-NMR experiments were automated with the ICON NMR using the acquisition and processing parameters (Appendix Table 1). The spectra were processed using Bruker Topspin 3.5 pl7.

\subsection{Statistical analysis}

At the end of the experiments, the rats were classified into gastric toxic or non-gastric toxic based on the presence or absence of any gastric toxicity, respectively. Spectra were bucketed to 0.04 ppm using AMIX software (BRUKER®, Germany). Water (4.9-4.7 ppm) and urea (6.1-5.5 ppm) regions were excluded. The bucket table was then imported to SIMCA 14.1 software (MKS Umetrics® Sweden). Skewed data were log-transformed. The data were then scaled using Pareto scaling (Pr scaling).

Principal component analysis (PCA) was conducted, and the score plot was examined to explore the behaviour of the data. Hotelling's T2 plot was used to discriminate intrinsic outliers in each group. The orthogonal partial least squares discriminant analysis (OPLSDA) was utilized to test the association between the buckets and gastric toxicity. This initial discriminatory model was the profiling model. The misclassification table was used to indicate the sensitivity, specificity, and accuracy of each model. The best differentiating model was selected based on the two goodness values, viz. the goodness-of-fit $\left(R^{2} Y\right)$ and goodness-of-prediction $\left(Q^{2} Y\right)$. A large $R^{2} Y$ (close to 1 ) is a necessary condition for a good model. It indicates good reproducibility. Likewise, a $\mathrm{Q}^{2} \mathrm{Y}$ value $>0.5$ signifies good predictivity. The variance between the two goodness values should not be too significant to ensure the right prediction and avoid overfitting. 
The variable importance for the projection (VIP) plot was then generated. The VIP plot summarizes the significance of the variables both to explain $X$ (the predictors) and to correlate to Y (the outcome). The value of VIP score, which is greater than 1 , is the typical rule for selecting variables that are important, relevant and potentially discriminating.[14, 15] Therefore, buckets with VIP value $>1$ were chosen for further analysis. These spectral buckets (with VIP values $>1$ ) were copied to an excel sheet and sorted in ascending order. The corresponding spectra for each bucket were verified in Topspin, and the spectral noise was excluded from true signals. The 3-(trimethyl-silyl) propionic acid (TSP) peak was defined as the reference, and the peaks were calibrated by reference to its peak.

A new data table was created by copying the relative integrals with their corresponding chemical shift (ppm) into an Excel sheet. The TSP integral was excluded from the table to not affect the analysis (as it is only a reference) before importing to SIMCA. The data was also log-transformed, Pareto scaled and explored initially using PCA. The OPLSDA was also applied, and VIP plots were generated. This second discriminatory model was the "Identification Model" (final model). The misclassification table was generated to show the proportion of correctly classified observations in the dataset. In SIMCA, the ability of a model to classify the individual subjects correctly or incorrectly is evaluated by the misclassification table tool.[16, 17]

Further, the permutation plot (Y-scrambling) was used as an internal validation of the model. This compares the goodness tests $\left(\mathrm{R}^{2}\right.$ and $\left.\mathrm{Q}^{2}\right)$ of the original model with the goodness test of several generated models by randomly permutating $Y$-observations (the outcome) while keeping X-matrix (the predictors) constant. The number of permutations was set to 100.[18] This means that the model was randomly built and validated 100 times. AUROC curve was also computed to visualize the performance of the discriminatory models. It serves as a quantitative measure of the performance of the model. The performance parameter ranges between 0.5 (bad classification) and 1.0 (perfect classification).

\subsection{Metabolites identification}

Metabolites were identified by systematically exploring three major databases, namely Biological Magnetic Resonance Data Bank (BMRB), Human Metabolome Database (HMDB) and Chenomx NMR Suite 6.0 (Chenomx® Inc., Canada). The quest begins by first exploring BMRB. The important chemical shifts identified from the identification model (previous step) were individually inputted in the designated field for exploring metabolites in the BMRB database. This generated several matching peaks along with their corresponding metabolites. The generated matching metabolites were individually cross-referenced in the HMDB database to ascertain their availability in the urine. The prospective metabolites available in the urine were further cross-matched in the Chenomx profiler to ascertain their identity.

\section{Conclusions}

Pharmacometabolomic analysis of the pre-dose ${ }^{1} \mathrm{H}-\mathrm{NMR}$ urine spectra identified metabolic signatures that correlated with the development of LDA-induced gastric toxicity and could predict gastric toxicity related to LDA. Citrate, hippurate, methylamine, trimethylamine N-oxide and alpha-keto-glutarate were the putative metabolites identified and possibly implicated in the LDA-induced gastric toxicity. The final model demonstrated good discriminatory properties, reproducibility and limited predictive capacity. This pharmacometabolomic approach can be translated to predict gastric toxicity in CAD patients when validated in humans.

Author Contributions: Conceptualization, A.S. and B.I.; methodology, A.S., N.A.K., C.E.S. and H.Z.; software, B.I. and C.H.T.; validation, B.I., H.Z. and N.A.K.; formal analysis, A.S., F.A.A. and M.M.; investigation, A.S., H.Z., N.A.K., F.A.A., C.E.S., C.H.T., M.M. and B.I.; resources, A.S. and B.I.; data curation, A.S., M.M. and B.I.; writing-original draft preparation, A.S.; writing - review and editing, A.S., H.Z., M.M. and F.A.A.; visualization, N.A.K., C.E.S., C.H.T. and H.Z.; supervision, B.I. 
and H.Z.; project administration, A.S., M.M., H.Z. and B.I. All authors have read and agreed to the published version of the manuscript.

Funding: This research received no external funding.

m

Data Availability Statement: Not applicable.

Acknowledgements: We want to thank Pharmacy Scholars Initiatives (PSI), formerly Young Pharmacist Scholars (YPS), for their support and mentorship.

Conflicts of Interest: The authors declare no conflict of interest.

Sample Availability: Samples of the compounds low-dose Aspirin are available from the authors.

\section{Appendix A}

Table A1: ${ }^{1} \mathrm{H}-\mathrm{NMR}$ experimental parameters.

\section{References}

1. Benjamin, E. J.; Virani, S. S.; Callaway, C. W.; Chamberlain, A. M.; Chang, A. R.; Cheng, S.; Chiuve, S. E.; Cushman, M.; Delling, F. N.; Deo, R.; de Ferranti, S. D.; Ferguson, J. F.; Fornage, M.; Gillespie, C.; Isasi, C. R.; Jiménez, M. C.; Jordan, L. C.; Judd, S. E.; Lackland, D.; Lichtman, J. H.; Lisabeth, L.; Liu, S.; Longenecker, C. T.; Lutsey, P. L.; Mackey, J. S.; Matchar, D. B.; Matsushita, K.; Mussolino, M. E.; Nasir, K.; O'Flaherty, M.; Palaniappan, L. P.; Pandey, A.; Pandey, D. K.; Reeves, M. J.; Ritchey, M. D.; Rodriguez, C. J.; Roth, G. A.; Rosamond, W. D.; Sampson, U. K. A.; Satou, G. M.; Shah, S. H.; Spartano, N. L.; Tirschwell, D. L.; Tsao, C. W.; Voeks, J. H.; Willey, J. Z.; Wilkins, J. T.; Wu, J. H.; Alger, H. M.; Wong, S. S.; Muntner, P., Heart Disease and Stroke Statistics-2018 Update: A Report From the American Heart Association. Circulation 2018, 137, (12), e67-e492.

2. Levine, G. N.; Bates, E. R.; Bittl, J. A.; Brindis, R. G.; Fihn, S. D.; Fleisher, L. A.; Granger, C. B.; Lange, R. A.; Mack, M. J.; Mauri, L., 2016 ACC/AHA Guideline Focused Update on Duration of Dual Antiplatelet Therapy in Patients With Coronary Artery Disease: A Report of the American College of Cardiology/American Heart Association Task Force on Clinical Practice Guidelines: An Update of the 2011 ACCF/AHA/SCAI Guideline for Percutaneous Coronary Intervention, 2011 ACCF/AHA Guideline for Coronary Artery Bypass Graft Surgery, 2012 ACC/AHA/ACP/AATS/PCNA/SCAI/STS Guideline for the Diagnosis and Management of Patients With Stable Ischemic Heart Disease, 2013 ACCF/AHA Guideline for the Management of ST-Elevation Myocardial Infarction, 2014 AHA/ACC Guideline for the Management of Patients With NonST-Elevation Acute Coronary Syndromes, and 2014 ACC/AHA Guideline on Perioperative Cardiovascular Evaluation and Management of Patients Undergoing Noncardiac Surgery. Circulation 2016, 134, (10), e123-e155.

3. Patrono, C., The multifaceted clinical readouts of platelet inhibition by low-dose aspirin. Journal of the American College of Cardiology 2015, 66, (1), 74-85.

4. 4. Sostres, C.; Lanas, A., Gastrointestinal effects of aspirin. Nature Reviews Gastroenterology and Hepatology 2011, 8, (7), 385-394.

5. Cryer, B.; Mahaffey, K. W., Gastrointestinal ulcers, role of aspirin, and clinical outcomes: pathobiology, diagnosis, and treatment. Journal of Multidisciplinary Healthcare 2014, 7, 137-146.

6. Clayton, T. A.; Lindon, J. C.; Cloarec, O.; Antti, H.; Charuel, C.; Hanton, G.; Provost, J.-P.; Le Net, J.-L.; Baker, D.; Walley, R. J.; Everett, J. R.; Nicholson, J. K., Pharmaco-metabonomic phenotyping and personalized drug treatment. Nature 2006, 440, (7087), 1073-7.

7. Silagy, C. A.; McNeil, J. J.; Donnan, G. A.; Tonkin, A. M.; Worsam, B.; Campion, K., Adverse effects of low-dose aspirin in a healthy elderly population. Clinical Pharmacology \& Therapeutics 1993, 54, (1), 84-89.

8. Li, J.-p.; Guo, J.-m.; Hua, Y.-q.; Zhu, K. Y.; Tang, Y.-p.; Zhao, B.-c.; Jia, L.-f.; Zhao, J.; Tang, Z.-s.; Duan, J.-a., The mixture of Salvia miltiorrhiza-Carthamus tinctorius (Danhong injection) alleviates low-dose aspirin induced gastric mucosal damage in rats. Phytomedicine 2016, 23, (6), 662-671.

9. Coen, M.; Goldfain-Blanc, F.; Rolland-Valognes, G.; Walther, B.; Robertson, D. G.; Holmes, E.; Lindon, J. C.; Nicholson, J. K., Pharmacometabonomic Investigation of Dynamic Metabolic Phenotypes Associated with Variability in Response to Galactosamine Hepatotoxicity. Journal of Proteome Research 2012, 11, (4), 2427-2440.

10. Ott, K.-H.; Aranibar, N., Nuclear magnetic resonance metabonomics: methods for drug discovery and development. Metabolomics: Methods and Protocols 2007, 247-271.

11. Szabo, S.; Trier, J. S.; Brown, A.; Schnoor, J.; Homan, H. D.; Bradford, J. C., A quantitative method for assessing the extent of experimental gastric erosions and ulcers. Journal of Pharmacological Methods 1985, 13, (1), 59-66.

12. Emwas, A.-H. M.; Salek, R.; Griffin, J.; Merzaban, J., NMR-based metabolomics in human disease diagnosis: applications, limitations, and recommendations. Metabolomics 2013, 9, (5), 1048-1072. 
13. Beckonert, O.; Keun, H. C.; Ebbels, T. M.; Bundy, J.; Holmes, E.; Lindon, J. C.; Nicholson, J. K., Metabolic profiling, metabolomic and metabonomic procedures for NMR spectroscopy of urine, plasma, serum and tissue extracts. Nature Protocols 2007, 2, (11), 2692-2703.

14. Tran, T. N.; Afanador, N. L.; Buydens, L. M. C.; Blanchet, L., Interpretation of variable importance in Partial Least Squares with Significance Multivariate Correlation (sMC). Chemometrics and Intelligent Laboratory Systems 2014, 138, 153-160.

15. Musharraf, S. G.; Siddiqui, A. J.; Shamsi, T.; Choudhary, M. I.; Rahman, A.-u., Serum Metabonomics of Acute Leukemia using Nuclear Magnetic Resonance Spectroscopy. Scientific Reports 2016, 6, 30693.

16. Blasco, H.; Błaszczyński, J.; Billaut, J. C.; Nadal-Desbarats, L.; Pradat, P. F.; Devos, D.; Moreau, C.; Andres, C. R.; Emond, P.; Corcia, P.; Słowiński, R., Comparative analysis of targeted metabolomics: Dominance-based rough set approach versus orthogonal partial least square-discriminant analysis. Journal of Biomedical Informatics 2015, 53, 291-299.

17. Caboni, P.; Liori, B.; Kumar, A.; Santoru, M. L.; Asthana, S.; Pieroni, E.; Fais, A.; Era, B.; Cacace, E.; Ruggiero, V.; Atzori, L., Metabolomics analysis and modeling suggest a lysophosphocholines-PAF receptor interaction in fibromyalgia. PloS One 2014, 9, (9), e107626-e107626.

18. Izquierdo-García, J. L.; Villa, P.; Kyriazis, A.; del Puerto-Nevado, L.; Pérez-Rial, S.; Rodriguez, I.; Hernandez, N.; RuizCabello, J., Descriptive review of current NMR-based metabolomic data analysis packages. Progress in Nuclear Magnetic Resonance Spectroscopy 2011, 59, (3), 263-270.

19. Szymańska, E.; Saccenti, E.; Smilde, A. K.; Westerhuis, J. A., Double-check: validation of diagnostic statistics for PLS-DA models in metabolomics studies. Metabolomics 2012, 8, (Suppl 1), 3-16.

20. Eriksson, L.; Kettaneh-Wold, N.; Trygg, J.; Wikström, C.; Wold, S., Multi-and megavariate data analysis: part I: basic principles and applications. Umetrics Inc: 2006.

21. Hua-Lin, C.; Li, H.-D.; Yan, X.-Z.; Sun, B.; Zhang, Q.; Yan, M.; Zhang, W.-Y.; Jiang, P.; Zhu, R.-H.; Liu, Y.-P.; Fang, P.-F.; Xu, P.; Yuan, H.-Y.; Zhang, X.-H.; Hu, L.; Yang, W.; Ye, H.-S., Metabolomic Analysis of Biochemical Changes in the Plasma and Urine of First-Episode Neuroleptic-Naïve Schizophrenia Patients after Treatment with Risperidone. Journal of Proteome Research 2012, 11, (8), 4338-4350.

22. Triba, M. N.; Le Moyec, L.; Amathieu, R.; Goossens, C.; Bouchemal, N.; Nahon, P.; Rutledge, D. N.; Savarin, P., PLS/OPLS models in metabolomics: the impact of permutation of dataset rows on the K-fold cross-validation quality parameters. Molecular BioSystems 2015, 11, (1), 13-19.

23. Westerhuis, J. A.; Hoefsloot, H. C.; Smit, S.; Vis, D. J.; Smilde, A. K.; van Velzen, E. J.; van Duijnhoven, J. P.; van Dorsten, F. A., Assessment of PLSDA cross validation. Metabolomics 2008, 4, (1), 81-89.

24. Golland, P.; Liang, F.; Mukherjee, S.; Panchenko, D. In Permutation tests for classification, International Conference on Computational Learning Theory, 2005; Springer: pp 501-515.

25. Baratloo, A.; Hosseini, M.; Negida, A.; El Ashal, G., Part 1: simple definition and calculation of accuracy, sensitivity and specificity. Emergency 2015, 3, (2), 48-49.

26. Takeuchi, K.; Ohishi, M.; Ota, S.; Suzumura, K.; Naraoka, H.; Ohata, T.; Seki, J.; Miyamae, Y.; Honma, M.; Soga, T., Metabolic Profiling to Identify Potential Serum Biomarkers for Gastric Ulceration Induced by Nonsteroid Anti-Inflammatory Drugs. Journal of Proteome Research 2013, 12, (3), 1399-1407.

27. Um, S. Y.; Park, J. H.; Chung, M. W.; Choi, K. H.; Lee, H. J., 1 H-Nuclear magnetic resonance-based metabolic profiling of nonsteroidal anti-inflammatory drug-induced adverse effects in rats. Journal of Pharmaceutical and Biomedical Analysis 2016, 129, 492-501.

28. Takeuchi, K.; Ohishi, M.; Endo, K.; Suzumura, K.; Naraoka, H.; Ohata, T.; Seki, J.; Miyamae, Y.; Honma, M.; Soga, T., Metabolomic analysis of the effects of omeprazole and famotidine on aspirin-induced gastric injury. Metabolomics 2014, 10, (5), 995-1004.

29. Wishart, D. S.; Feunang, Y. D.; Marcu, A.; Guo, A. C.; Liang, K.; Vazquez-Fresno, R.; Sajed, T.; Johnson, D.; Li, C.; Karu, N.; Sayeeda, Z.; Lo, E.; Assempour, N.; Berjanskii, M.; Singhal, S.; Arndt, D.; Liang, Y.; Badran, H.; Grant, J.; Serra-Cayuela, A.; Liu, Y.; Mandal, R.; Neveu, V.; Pon, A.; Knox, C.; Wilson, M.; Manach, C.; Scalbert, A., HMDB 4.0: the human metabolome database for 2018. Nucleic Acids Research 2018, 46, (D1), D608-d617.

30. Wang, Z.; Klipfell, E.; Bennett, B. J.; Koeth, R. A.; Levison, B. S.; Dugar, B.; Feldstein, A. E.; Britt, E. B.; Fu, X.; Chung, Y. M.; Wu, Y.; Schauer, P.; Smith, J. D.; Allayee, H.; Tang, W. H.; DiDonato, J. A.; Lusis, A. J.; Hazen, S. L., Gut flora metabolism of phosphatidylcholine promotes cardiovascular disease. Nature 2011, 472, (7341), 57-63.

31. Um, S. Y.; Park, J. H.; Chung, M. W.; Kim, K.-B.; Kim, S. H.; Choi, K. H.; Lee, H. J., Nuclear magnetic resonance-based metabolomics for prediction of gastric damage induced by indomethacin in rats. Analytica Chimica Acta 2012, 722, 87-94 